\title{
A Novel Organometallic Macrocycle Based on Half-sandwich Ruthenium Motif
}

Xiu-Xiu Li, Qing-Ya He, Fanghui Wu, Guozan Yuan, and Wei-Bin Yu*

Analysis and Testing Central Facility, School of Chemistry and Chemical Engineering, Anhui University of Technology, Maanshan 243002, China

\begin{abstract}
The rational design and construction of a novel trinuclear macrocycle based on half-sandwich ruthenium was herein elaborated in detail, and consequently, fully characterized by 1D NMR, IR, EA and single crystal X-ray analysis, revealing its specific molecular structure which was a classic three-member ruthenium ring bridged by an organic linker [(E)-2-(2-(pyridin-4-yl)vinyl)quinolin-8-ol (HL)]. To further examine its photo and electronic properties, the mothed of UV-vis and CV was carried out at ambient temperature, revealing it has a good application for catalyzing water oxidation under alkaline conditions.
\end{abstract}

Keywords: Half-sandwich ruthenium; Macrocycle; Cyclic voltammetry; Characterization; Electronic properties; Organic linker.

Corresponding author. Tel: +86 555 2311087. fax: +86 5552311087 E-mail address: yuweibin@ahut.edu.cn. 


\section{Introduction}

The directional bonding approach, which is one of the three main branches for constructing the supramolecular architectures, is a general, high-yielding synthetic strategy that gives access to a wide variety of $2 \mathrm{D}$ and $3 \mathrm{D}$ supramolecular ensembles[1]. Since Fujita and Stang's group reported that the rational syntheses of molecular squares, metallamarcocycles, and metallacages were assembled using this design strategy in the early 1990[2-4], it has grown into a mature strategy of allowing for a combinatorial molecular library consisting of complementary building blocks in the past decades[5,6]. According to pioneering work, Prof. Stang summarized two basic structural requirements for the construction of supramolecular architectures by the directional bonding approach as following: 1) the complementary precursor units must be structurally rigid with predefined bite angles; and 2) the appropriate stoichiometric ratio of the precursors must be used. In terms of the requirements, the donor building blocks must have two or more binding sites possessing angular orientations ranging from $0^{\circ}$ to $180^{\circ}$.

In light of aforementioned requirements, the receptors which are general metal-containing subunits are vital importance in this design approach due to its available coordination sites which are at a fixed angle relative to one another for binding incoming ligands[1]. Half-sandwich compounds of $\mathrm{Cp} * \mathrm{M}\left(\mathrm{M}=\mathrm{Ir}, \mathrm{Rh}\right.$; $\mathrm{Cp}^{*}=$ pentamethylcyclopentadienyl) and (cymene)Ru[7-9], whose geometry is a classic three-leg-piano-stool coordination site born three donors (general $\mathrm{N}$ and $\mathrm{O}$ atoms from organic linkers) to construct $2 \mathrm{D}$ and $3 \mathrm{D}$ organometallic complexes, are suitable for constructing supramolecular architectures to meet the above requirements. The first case is that using half-sandwich fragment to construct rhodium-DNA/RNA compounds was reported by Fish[10]. The field of using half-sandwich fragments based on cymeneRu to self-assemble organometallic complexes has become mature, since the group of Severin reported a receptor for small cations and anions[11,12]. The fascination with physical and chemical properties of these organometallic complexes on basis of half-sandwich ruthenium fragments has led us to enter this area with a desire to design specific ensembles. 
On the other hand, the necessary effect of organic ligands is not ignored in the field of bonding to metal centers to construct supramolecular units. Among these candidates, containing $\mathrm{N}$ and $\mathrm{O}$ atoms of organic linkers utilized as donors are widely and progressively developed with many disciplines for constructing aesthetically pleasing organometallic structures[13-18, 26-29]. Robustness, rigidity, and suitable geometry of over two donors of these organic linkers are necessary to construct an elegant and intricate functional structure of organometallic macrocycles. A difunctional pyridyl-substituted 8-hydroxyquinoline ligand is promisingly meeting the requirements. The groups of Lewiński and Yuan have reported that using 8-hydroxyquinoline-based organic ligands as linkers to assemble plethoric metal-orgnaic frameworks exhibiting unusual optical properties[19-22]. This inspired us to use the analogues to rational design and controllable synthesize metallacycles based on half-sandwich iridium, rhodium and ruthenium fragments.

\section{((Insert Chart 1 here))}

In this work, we use (E)-2-(2-(pyridin-4-yl)vinyl)quinolin-8-ol (HL) (Chart 1) as an organic linker to construct trinuclear metallacycles based on cymeneRu motif under mild conditions. The specific elaboration of its structural characterization is confidently proved by NMR and X-ray crystallographic analysis exhibiting an aesthetic architecture.

\section{Experimental}

\subsection{General conditions}

General. All solvents and reagents were of commercial grade. The starting materal of $\left[\mathrm{CyRuCl}_{2}\right]_{2}$ and organic ligand $\mathrm{HL}$ were synthesized according to the previous literature[23,24]. UV-vis spectra were collected using a Varian Cary 50 Bio UV-vis spectrometer with samples in a $1.0 \mathrm{~cm}$ quartz cuvette at $\mathrm{pH} 7.0$. Elemental analyses were performed on an Elementar III Vario EI analyzer. IR spectra was measured on a Nicolet Avatar-360 spectrophotometer (as KBr pellet). Cyclic voltammograms (CVs) were measured on Electrochemical Worksatation (CHI660E). The crystal sample used for X-ray crystallography was obtained by diffusion of ether into methanol solution of complex 1 at room temperature. 


\subsection{The synthesis of the complex 1}

$\left[\mathrm{CyRuCl}_{2}\right]_{2}(62.0 \mathrm{mg}, 0.1 \mathrm{mmol})$ was dissolved in $10.0 \mathrm{~mL}$ of methanol at room temperature. Then, the solution was added into 4 equiv of $\mathrm{AgNO}_{3}(68.0 \mathrm{mg}, 0.4 \mathrm{mmol})$ in dark and stirred vigorously for 6 hours. After then, the reaction solution was added to about $0.1 \mathrm{mmol}(35.0 \mathrm{mg})$ of $\mathrm{HL}$ kept vigorously stirring for 12 hours, red precipitate collected by vacuum filtration in air. 1, Yield, $47.4 \mathrm{mg}, 71 \%$.

Complex 1: ${ }^{1} \mathrm{H}$ NMR (400 MHz, $\left.d_{2}-\mathrm{CD}_{2} \mathrm{Cl}_{2}, \mathrm{ppm}\right): \delta 1.2$ (d, 6H, $\left.\mathrm{CH}_{3}-\mathrm{Cymene}\right), 2.3$ (s, 3H, $\mathrm{CH}_{3}$-Cymene), 2.53 (m, 1H, CH-Cymene), 7.07 (s, 2H, Cymene), 7.10 (d, 2H, Cymene), 7.57 (d, 1H, Vinyl), 7.60 (d, 1H, Vinyl), 6.89 (d, 1H, Quinolin-8-ol), 7.92 (d, 2H, Pyridyl), 8.02(d, 1H, Quinolin-8-ol), 8.07 (s, 1H, Quinolin-8-ol), 8.13(d, 1H, Quinolin-8-ol), 8.66(d, 2H, Pyridyl). ${ }^{13} \mathrm{C} \mathrm{NMR}\left(100.6 \mathrm{MHz}, d_{2}-\mathrm{CD}_{2} \mathrm{Cl}_{2}, \mathrm{ppm}\right): \delta$ 21.15( $\mathrm{CH}_{3}$-Cymene $), \quad 23.85\left(\mathrm{CH}_{3}\right.$-Cymene $), \quad 33.65(\mathrm{CH}$-Cymene $), \quad 126.18$ (Benzyl-Cymene), 128.85(Benzyl-Cymene), 135.01(Benzyl-Cymene), 144.44(Benzyl-Cymene), 112.44 (Quinolin-8-ol), 116.07 (Quinolin-8-ol), 120.22 (Quinolin-8-ol), 124.27(Quinolin-8-ol), 128.99(Quinolin-8-ol), 135.14(Quinolin-8-ol), 145.88 (Quinolin-8-ol), 150.37(Quinolin-8-ol), 155.14(Quinolin-8-ol), 130.31 (Vinyl) 133.03(Vinyl), 126.62(Pyridyl), 167.60(Pyridyl), 168.37(Pyridyl). Elemental analysis calced (\%) for $\mathrm{C}_{84} \mathrm{H}_{93} \mathrm{AgN}_{11} \mathrm{O}_{21} \mathrm{Ru}_{3}: \mathrm{C}, 50.35$. H, 4.68. N, 7.69. Found: C, 50.28. H, 4.59. N, 7.61. IR(KBr, $\left.\mathrm{cm}^{-1}\right): 1390(v, C=O)(\mathrm{s})$.

\subsection{X-ray crystal structure determinations}

Data were collected on a CCD-Bruker SMART APEX system. All the determinations of unit cell and intensity data were performed with graphite-monochromated MoK $\alpha$ radiation $(\lambda=0.71073 \AA)$. All the data were collected at room temperature using the $\omega$ scan technique. The structure was solved by direct methods, using Fourier techniques, and refined on $F^{2}$ by a full-matrix least-squares method. All the calculations were carried out with the SHELXTL program[25]. A summary of the crystallographic data and selected experimental information are given in supporting information.

\section{Results and discussion}

3.1 Synthesis considerations and spectroscopic characterization 
By directional bonding approach of constructing supramolecular ensembles, the one-pot methodology of a synthetic procedure is oftentimes employed with a mature strategy in assembling combinatorial metallamacrocycles consisting of complementary building blocks. This using (E)-2-(2-(pyridin-4-yl)vinyl)quinolin-8-ol (HL) as incoming ligand which is structurally rigid with predefined bite angles, directly combine a trinuclear metallamacrocycle with an appropriate stoichiometric ratio of half-sandwich ruthenium fragment as metal-containing subunits. $\left[\mathrm{CyRu}(u-\mathrm{Cl})_{2} \mathrm{Cl}_{2}\right]$ as starting material was treated with silver salt and then mixed with $\mathrm{HL}$ forming a trinuclear metallamacrocycle $\left[(\mathrm{CyRuL})\left(\mathrm{NO}_{3}\right)\right]_{3}\left(\mathrm{AgNO}_{3}\right) \mathbf{1}$ with chelating Ru-N,O coordination(Scheme 1). The specie was fully characterized with a host of NMR and EA experiments to confirm its stoichiometries. X-ray crystallography was also used to gain further structural insight on a suitable crystal obtained by diffusion ether into the methanol solution of the macrocycle for a week.

\section{((Insert Scheme 1 here))}

1D NMR analysis of the complex 1 supported the formation of discrete homoleptic metallamacrocycle with highly symmetric geometry. The 1H NMR spectra of 1 exhibited sharp peaks ( $\delta 1.20$ for $\mathrm{CH}_{3}$-Cymene, 2.3 for $\mathrm{CH}_{3}-\mathrm{Cymene}$ and 2.53 for $\mathrm{CH}-\mathrm{Cymene}$ with ratio of $6: 3: 1)$ with concomitant ${ }^{13} \mathrm{C}$ satellites corresponding to a cymene group. The H protons on the pyridine rings (7.92 and $8.66 \mathrm{ppm}$, respectively) were split into sets of two doublets upon metal-coordination, which was consistent with the previous work[24]. As 1D NMR spectra depicted, the protons of naphthalene group relative to those of $\mathbf{1}$ were also observed with complicated signals with a logical ratio of $1: 1: 1: 1: 1$. The well-defined signals in 1D NMR spectra as well as the good solubility of the complex support the formation of a discrete structure as a sole ensemble with highly symmetry.

\section{((Insert Figure 1 here))}

\subsection{Crystal structure description}

The trinuclear homoleptic geometry of this metallamacrocycle was distinctly classified by X-ray crystallography. The cationic framework of this complex consists of three cymeneRu fragments and organic ligands (Figure 1). Each metal center with 
cis-capped by cymene rings bears two quinolin-8-ol-based ligand with $(\mathrm{N}, \mathrm{O})$ and $\mathrm{N}$ coordination, exhibiting a classic three-piano-leg-stool geometry with $\mathrm{C}_{3 h}$ axis symmetry. The adjacent organic linkers build up a distorted triangle with three length of edges $9.61 \AA$ and three angles of $60^{\circ}$, suggesting a regular geometry.

The triangle in the cationic framework capture one nitrate and are surrounded by three nitrate radicals and one silver ion combining a holonomic tetrahedron (Figure 2a). Due to the defective tetrahedral geometry, the channel (Figure 2c) and the bulk material (Figure 2b) are constructed by one molecule of the metallamacrocycles in layer-by-layer mode.

\section{((Insert Figure 2 here))}

\subsection{Optic chemistry}

UV-visible absorption spectroscopy was first technique used to monitor UV absorption of the metallamacrocycles. This method was able to detect the coordination changes because of the donors and coordination centers interactions that, for example, result from quinolin-8-ol bonding to half-sandwich ruthenium fragments in the trinuclear triangular conformation. Furthermore, the techniques enabled highly incisive structural investigations that can be employed to study dynamics in the coordination chemistry[24]. Herein, we thus performed UV-visible spectrophotometer on complex 1 to evaluate their electronic structures, affording a characteristic peak at $338 \mathrm{~nm}$ coupled with a broad absorption band centered at $526 \mathrm{~nm}$ (Figure 3). In addition, a set of the feature peak in high energy region was characteristic of $\pi-\pi^{*}$ transition based on 8-hydroxyquinoline ligand, and the band in visual region could be attributed to the MLCT derived from cymeneRu moiety.

\section{((Insert Figure 3 here))}

\subsection{Electronic properties}

In the present study, cyclic voltammetry was carried out to determine the electronic properties of the metallamacrocycle with a complementary simple model to oredict the curves, exhibiting a broad, strongly concentration-dependent peak center at $1.0 \mathrm{~V}$ (vs $\mathrm{Ag} / \mathrm{AgCl}$ ) (Figure 4). As a result, the persuasive observation strongly proved the 
complex might be utilized as precatalyst for water oxidation in the alkaline conditions.

\section{((Insert Figure 4 here))}

\section{Conclusion}

In this context, we described in detail the formation and physical properties of a novel metallamacrocycle based on half-sandwich ruthenium born by a quinolin-8-ol-based organic linker. An elegant and aesthetic trinuclear distorted triangle geometry was unambiguously confirmed by fully characterization with NMR and X-ray crystallography. Furthermore, the evaluation of its optical and electronic properties was employed UV-visible absorption spectroscopy and cyclic voltammetry, indicating distinguishing interaction between coordination ligand and half-sandwich ruthenium fragment, and fascinating potential application in the field of catalyzing water oxidation. In the future, we will endeavor exploring its application in the optical and catalytic field.

\section{Acknowledgements}

This work was supported by the National Science Foundation of China (21301003, 21271006) and Anhui Provincial Science Foundation (1408085QB30). 


\section{References}

[1]R. Chakrabarty, P. S. Mukherjee, P. J. Stang, Chem. Rev., 111 (2011) 6810.

[2]M. Fujita, J.Yazaki, K. Ogura, J. Am. Chem. Soc., 112 (1990) 5645.

[3]M. Fujita, J. Yazaki, K. Ogura, Chem. Lett., (1991) 1031.

[4]P. J. Stang, D. H. Cao, J. Am. Chem. Soc., 116 (1994) 4981.

[5]T. R. Cook, Y.-R. Zheng, P. J. Stang, Chem. Rev., 113 (2013) 734.

[6]T. R. Cook, P. J. Stang, Chem. Rev., 115 (2015) 7001.

[7]Y.-F. Han, W.-G. Jia, W.-B. Yu, G.-X. Jin, Chem. Soc. Rev., 38 (2009) 3419.

[8]A. K. Singh, D. S. Pandey, Q. Xu, P. Braunstein, Coord. Chem. Rev., 270-271

(2014) 31 .

[9] Y.-F. Han, G.-X. Jin, Acc. Chem. Res., 47 (2014) 3571.

[10]R. H. Fish, Coord. Chem. Rev., 185-186 (1999) 569.

[11]K. Severin, Coord. Chem. Rev., 245 (2003) 3.

[12]K. Severin, Chem. Commun., (2006) 3859.

[13]H. Li, Z.-J. Yao, D. Liu, G.-X. Jin, Coord. Chem. Rev. (2015) 139.

[14]L. Zhang, Y.-J. Lin, Z.-H. Li, G.-X. Jin, J. Am. Chem. Soc., 137 (2015) 13670.

[15]Y.-Y. Zhang, X.-Y. Shen, L.-H. Weng, G.-X. Jin, J. Am. Chem. Soc., 136 (2014) 15521.

[16]S.-L. Huang, Y.-J. Lin, Z.-H. Li, G.-X. Jin, Angew. Chem. Int. Ed., 53 (2014) 11218.

[17]H. Li, Y.-F. Han, Y.-J. Lin, Z.-W. Guo, G.-X. Jin, J. Am. Chem. Soc., 136 (2014) 2982.

[18]Z.-J. Yao, W.-B. Yu, Y.-J. Lin, S.-L. Huang, Z.-H. Li, G.-X. Jin, J. Am. Chem. Soc., 136 (2014) 2825.

[19]K. Sokołowski, W. Bury, I. Justyniak, D. Fairén-Jiménez, K. Sołtys, D. Prochowicz, S. Yang, M. Schröderd, J. Lewiński, Angew. Chem. Int. Ed., 52 (2013) 13414.

[20]T. Kaczorowski, I. Justyniak, D. Prochowicz, K. Zelga, A. Kornowicz, J. Lewiński, Chem. Eur. J., 18 (2012) 13460.

[21]K. Sokołowski, I. Justyniak, W. Śliwiński, K. Sołtys, A. Tulewicz, A. Kornowicz, 
R. Moszyński, J. Lipkowski, J. Lewiński, Chem. Eur. J., 18 (2012) 5637.

[22]G. Yuan, Y. Huo, X. Nie, H. Jiang, B. Liu, X. Fang, F. Zhao, Dalton Trans., 42 (2013) 2921.

[23]C. White, A. Yates, and P. M. Maitles, Inorg. Synth., 29 (1992) 228.

[24]G. Yuan, G. Hu, W. Shan, S. Jin, Q. Gu, J. Chen, Dalton Trans., 44 (2015) 17774.

[25]G. M. Sheldrick, SHELXL-97. Universität Göttingen: Germany, 1997.

[26]Y.-F. Han, L. Zhang, L.-H. Weng, G.-X. Jin, J. Am. Chem. Soc. 136 (2014) 14608.

[27]Y.-F. Han, G.-X. Jin, Chem. Soc. Rev. 43 (2014) 2799.

[28]W.-Y. Zhang, Y.-F. Han, L.-H. Weng, G.-X. Jin, Organometallics, 33 (2014) 3091.

[29]Y.-F. Han, H. Li, L.-H. Weng, G.-X. Jin, Chem. Comm. 46 (2010) 3556. 
Figure captions:

Chart 1. Schematic structure of organic ligand (HL).<smiles>Oc1cccc2ccc(/C=C/c3ccncc3)nc12</smiles>

HL

Scheme 1. Synthetic process of macrocycle 1.
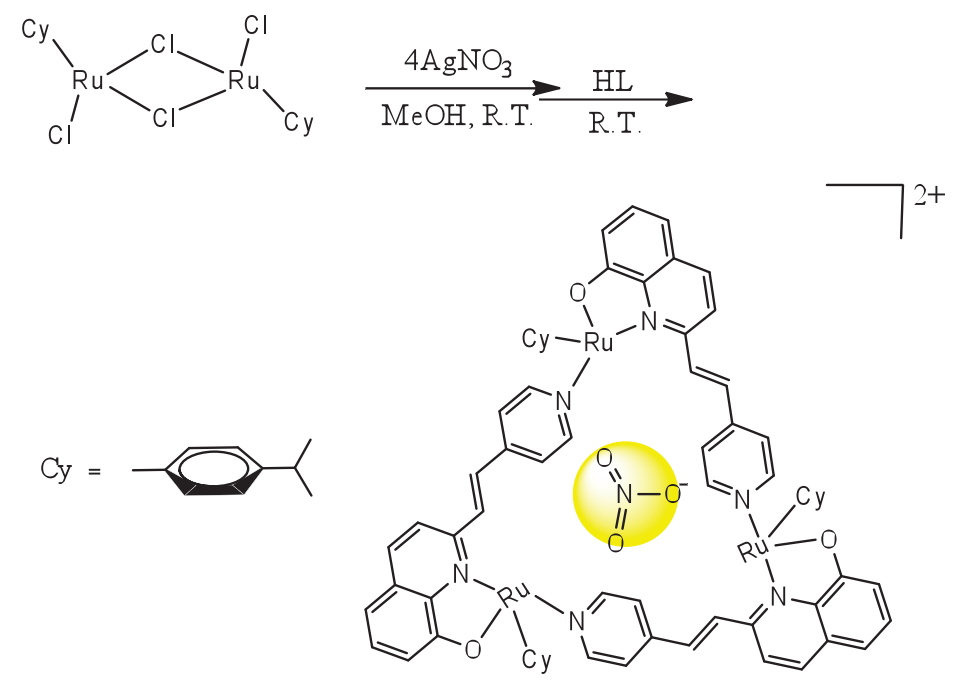
Figure 1. ORTEP drawing of cationic structure in the macrocycle. $\mathrm{H}$ atoms and anions were omitted for clarity. Selected bonds $(\AA)$ and $\operatorname{angles}\left({ }^{\circ}\right)$ : $\operatorname{Ru}(1)-\mathrm{O}(1)=2.049(5)$, $\mathrm{Ru}(1)-\mathrm{N}(3)=2.126(6), \quad \mathrm{Ru}(1)-\mathrm{N}(2)=2.132(6) ; \mathrm{O}(1)-\mathrm{Ru}(1)-\mathrm{N}(3)=78.9(2)$, $\mathrm{O}(1)-\mathrm{Ru}(1)-\mathrm{N}(2)=87.5(2), \mathrm{N}(3)-\mathrm{Ru}(1)-\mathrm{N}(2)=87.2(2)$.

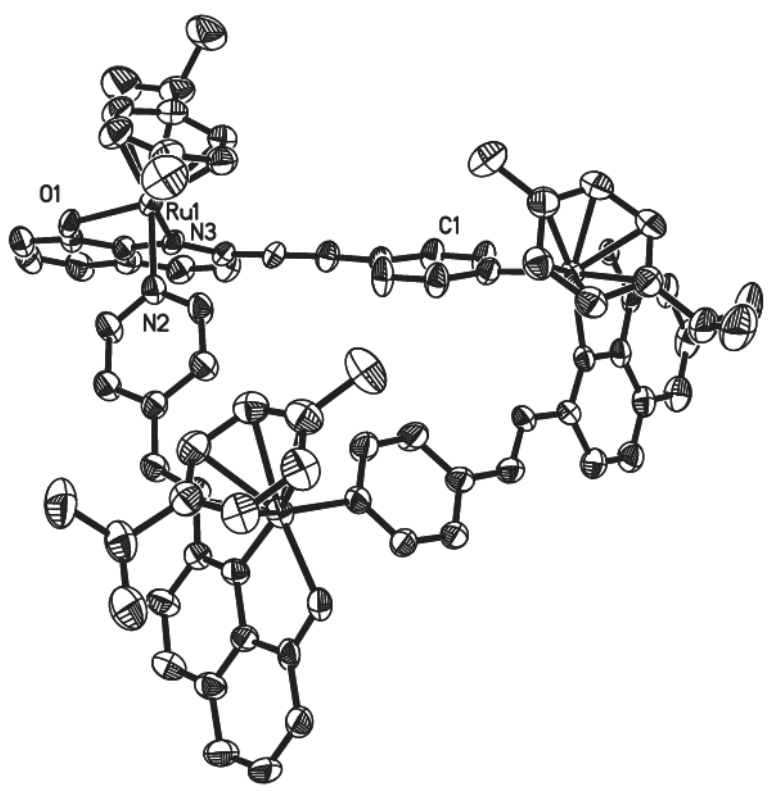

Figure 2. a) molecular structures of 3 ; b) packing-mode structures in 3 ; c) nanochannel architectures for 3. $\mathrm{H}$ atoms were omitted for clarity. Dark red, $\mathrm{Ru}$; lighting green, $\mathrm{Cl}$; red, $\mathrm{O}$; blue, $\mathrm{N}$; gray, $\mathrm{C}$.

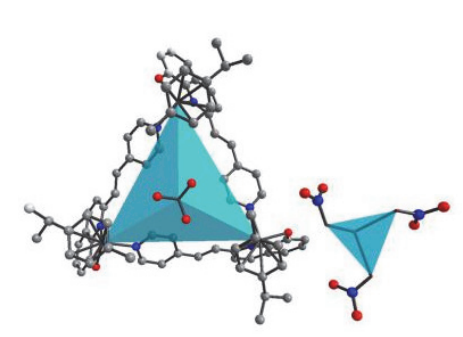

a




Figure 3. UV-vis curve of the Ru-based macrocycle.

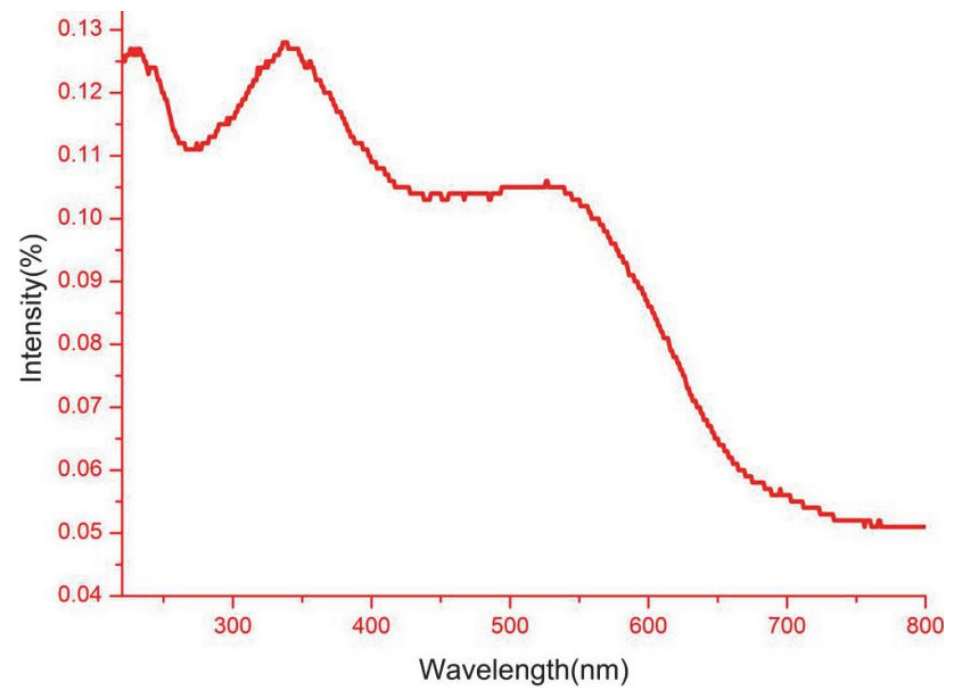

Figure 4. The curves of cyclic voltammetry for based on the macrocycle. (Conditions: $[0.1 \mathrm{M}] \mathrm{PBS}$ as alkaline solutions with $\mathrm{pH}=9.0$ at room temperature; scan rate: 50 $\mathrm{mV} / \mathrm{s})$

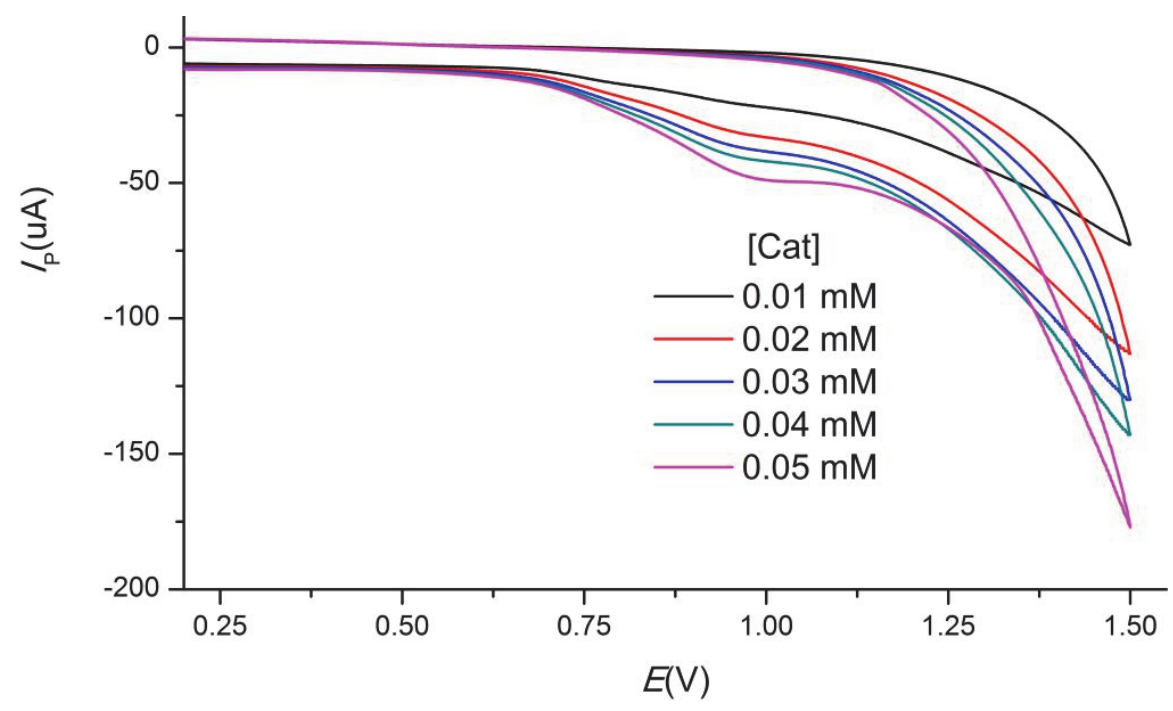

